\title{
Consumo de drogas en adolescentes. Una reflexión conceptual
}

\section{Drug consumption in adolescents. A conceptual reflection}

DOI: http://dx.doi.org/10.17981/cultedusoc.10.1.2019.04

Recibido: 25/09/2018 Aceptado: 14/02/2019

\author{
Oscar Armando Erazo Santander \\ Fundación Universitaria de Popayán (Colombia) \\ oscar.erazosantander@gmail.com.
}

Para citar este artículo:

Erazo, O. (2019). Consumo de drogas en adolescentes. Una reflexión conceptual. Cultura, Educación y Sociedad, 10(1). 53-66. DOI: http:// dx.doi.org/10.17981/cultedusoc.10.1.2019.04

\section{Resumen}

El artículo de reflexión aborda en una dimensión conceptual el tema de las drogas, consecuencias, intervención y propuesta de análisis. Se generan cuatro apartados argumentativos, discriminados así: El primer apartado explica las drogas y prevalencia, refiriendo el incremento del $60 \%$ de consumidores en Sur América y la existencia de un $63 \%$ de estudiantes en Colombia que mencionan haber consumido una droga. El segundo describe la acción de las drogas en el sistema dopaminergico y su afectación en el sistema nervioso central, además de otras afectaciones enfatizando las políticas de estado para atender la situación problema. El tercer cuerpo del artículo explica la demanda a través de dos modelos, uno muestra el consumo, funcionamiento y consecuencias de tipo neurológico y el otro busca comprender los antecedentes de la decisión y acción de consumo, con resultados coherentes, pero no concluyentes. Con base a lo expuesto se explica la elección y consumo de drogas, a través de la integración de una tesis cognitiva como la intencionalidad, explicada por la teoría de conducta planificada y la tesis neuropsicológica con funciones ejecutivas asociadas a la toma de decisión, planeación e inhibición. El cuarto apartado refiere la vulnerabilidad en la adolescencia para realizar elecciones y planear metas de corto plazo con desventaja futura y se plantea la posibilidad que la intención negativa y la alta madurez en Funciones Ejecutivas (FE) llevarían a la inhibición de elección y consumo a diferencia la intención positiva y baja madurez de $\mathrm{FE}$ que concluirían en elecciones y consumo de tendencia positiva.

Palabras clave: Elección de consumo de drogas; adolescentes; drogas

\begin{abstract}
The reflection article addresses in a conceptual dimension the issue of drugs, consequences, intervention and proposed analysis. It generates four argumentative sections, which are discriminated against in this way: The first section explains drugs and prevalence, referring to the $60 \%$ increase in the number of consumers in South America and the existence of $63 \%$ of students in Colombia who mention having consumed a drug. The second describes the action of drugs in the dopaminergic system and their affectation in the central nervous system, in addition to other affectations emphasizing state policies to address the problem situation. The third body of the article explains the demand through two models, one shows consumption, functioning and neurological consequences and the other seeks to understand the antecedents of the decision and action of consumption, with coherent results, but not conclusive. Based on the above, the choice and consumption of drugs is explained through the integration of a cognitive thesis such as intentionality, explained by the theory of planned behavior and the neuropsychological thesis with executive functions associated with decision making, planning and inhibition. The fourth section refers to the vulnerability in adolescence to make choices and plan short-term goals with future disadvantage and raises the possibility that negative intention and high maturity in Executive Functions (FE) would lead to inhibition of choice and consumption as opposed to positive intention and low maturity of $\mathrm{FE}$ that would lead to positive trend choices and consumption.
\end{abstract}

Keywords: Choice of drug use; adolescent; drug use. 


\section{INTRODUCCIÓN}

Drogas y consumo, prevalencia de una pandemia

El consumo de drogas es considerado actualmente una pandemia mundial, de difícil prevención e intervención y con posibilidades de generar patrones clínicos de dependencia y abuso que afectan la dimensión personal, familiar, social, económica y política de la sociedad (Becoña, 2002; Corominas, Roncero, Bruguera y Casas, 2007; MinSalud, 2007). En el 2015 existían 324 millones de consumidores, de los cuales, 39 millones eran clasificados en dependencia y abuso (Echavarria y Giraldo, 2015; Pérez, Mejía, Reyes y Cardozo, 2015). En Suramérica para el 2010 aumenta al $60 \%$ los consumidores con mayor numero en adolescentes, necesitando intervención clínica 2.4 millones de personas (Uribe y Castaño, 2014; Melo y Casthaneria, 2010; Toaza, 2011). Como se refleja en estos indicadores la situación se torna cada vez más compleja, por cuanto es un problema que se ha ido generalizando a nivel mundial con serias consecuencias en la salud integral del individuo.

Para el 2001 Estados Unidos describía a 70.9 millones como adictos al tabaco con iniciación a los 12 años (National Institute on Drug Abuse-NIDA, 2010a) en España, el 87\% de sujetos entre 15 a 18 años eran fumadores (Lloret, MorellGomis, Laguia y Moriano, 2017) y en el mundo existían 1.200 millones con proyección a 2000 millones para el 2030 (Becoña, 2002). 730.000 sujetos en el 2008 eran adictos a los inhalantes con pico a los 14 años, siendo el popper el producto más consumido en Estados Unidos (NIDA, 2011), el cannabis cuenta con
19.8 millones de consumidores, de los cuales el $81 \%$ son adolescentes en Estados Unidos (NIDA, 2015), en España el $12 \%$ de jóvenes de 15 hasta adultos de 34 años la consume, al igual que uno de cada cuatro adolescentes escolarizados (Lloret, et al. 2017; Martínez y Ojeda, 2016).

Existen 359.000 consumidores de crack y uno de seis estudiantes consume cocaína en Estados Unidos (Hidalgo y Redondo, 2005; NIDA, 2009) un 10\% en España (Lloret, et al. 2017) y existen 11 millones de consumidores de éxtasis o MDMA (NIDA, 2007b; 2010b) en España es el 6\% de jóvenes entre 15 a 18 años (Castellanos y Espinosa, 2013).

En Colombia para el 2013 habían ingresado 480.000 menores a urgencias por adicción y en el 2015 un $63 \%$ de estudiantes de 6 a 11 habían consumido alguna droga y eran consumidores de tabaco un $24.3 \%$, marihuana el $7 \%$, cocaína el $2.8 \%$, bazuco $0.7 \%$, inhalantes $3.1 \%$, éxtasis $1.2 \%$, ácidos $1.5 \%$, tranquilizantes $1.1 \%$, popper $2.5 \%$, heroína $0.5 \%$ y dick (dicloruro de metileno) el 4.1\%. (Andrade, Betancourt, Moreno y Alvis, 2017; Pérez, et al. 2015) y se clasifican en dependencia de cannabis 250 mil casi el $57 \%$ de los consumidores de esta droga, $140 \mathrm{mil}$ de cocaína siendo el $60 \%$ consumidor de esa droga y 34 mil de bazuco siendo el $78 \%$ (Ministerio de la protección social y salud, 2008).

La existencia de las drogas y su preocupación de consumo en los adolescentes de Sur América y Colombia, es evidente y permite plantear preguntas reflexivas que quizás pueda abrir la comprensión de la problemática, ¿por qué el temor en el consumo de drogas? y si el temor es justificado ¿qué se ha hecho para mejorar la problemática? 
Drogas, consecuencias e intervención (un problema político)

La droga es un concepto genérico, denominado así, por su capacidad para generar respuestas con recompensa, producto de la activación biológica y neurológica del sistema nervioso central ante la sustancia (Organización Mundial de la Salud-OMS, 2005; Asociación Psiquiátrica AmericanaAPA, 2014). La sensación de placer y bienestar tiene costos en la vida de los sujetos. Uno de ellos es la ausencia de control en el consumo.

La pérdida de control implica el pensamiento obsesivo y el comportamiento compulsivo por consumir la droga a pesar de sus consecuencias. Hecho que no es producto de la función consiente, si no de la modificación biológica en el Sistema Nervioso Central (SNC) y que se conoce como dependencia y abuso de sustancias psicoactivas (Alcázar, Verdejo, Bouso y Ortega, 2015; APA, 2014; NIDA, 2012; Martínez, 2012; Patiño, 2012; Sánchez, Arroyo y Azcona, 2014).

Con el consumo frecuente los sistemas de comunicación caracterizados por la regulación de neurotransmisores y receptores operados molecularmente, son modificados llevándolos a su eliminación, inhibición o aumento (como sucede con receptores de dopamina y serotonina) (Quintero, 2015). La eliminación o inhibición de receptores termina por desconectar regiones cerebrales, como sucede con las conexiones entre el sistema límbico y la corteza prefrontal. Esta desconexión inactiva la corteza prefrontal (CPF) entregando el control a la corteza subcortical encargada de mecanismos y comportamientos automáticos, reflejos y no consientes (Ruiz-Sánchez, Pedrero-Pérez, Lozoya-Delgado, Llanero-Luque, Rojo-Mota y Puerta-García, 2012).
La irregularidad en la CPF afecta el funcionamiento ejecutivo describiendo deficiencias en la atención, memoria de trabajo, flexibilidad, planeación y resolución de problemas y los daños con la conexión subcortical lleva a desordenes afectivos, conductuales y sociales caracterizados por trastornos depresivos, tendencia suicida, dificultades al dormir, comportamiento impulsivo, hiperactivo, agresivo y tendencia disocial (Buela-Casal, RiañoHernández y Guillen, 2015).

La alta estimulación de la droga a centros de recompensa, sistema dopaminergico o dopamina, lleva al desarrollo de múltiples conexiones que activan frecuentemente áreas subcorticales y componentes como el núcleo accumbens, tallo cerebral o núcleos activadores de Neurotrasmisores (NT) relacionados con la sensación de placer y los que no son controlados por la CPF en especial el dorso lateral. $\mathrm{Su}$ consecuencia se expresa en toma de decisiones impulsivas, desprovista de juicio moral o riesgosas como sexualidad sin protección (VIH, hepatitis C, embarazo no deseado, ETS) (Méndez, 2012; OMS, 2005), conducción de automóviles (10\% de muertes relacionados con drogas y accidentes de tránsito) (Balogun, et al. citado por Pérez, et. al 2015) o realización de conductas extremas de tipo negativista -desafiante o disocial-, como lo indica Pérez-Gómez citado por Fuenmayor y Saavedra, 2015) que, en una muestra de 1667 menores infractores y 9.500 adolescentes escolarizados no infractores, encontró que los infractores eran consumidores con frecuencia 20 veces superior, consumiendo el $75 \%$ tranquilizantes, $50 \%$ cocaína, 36\% inhalables, $21 \%$ bazuco, $12 \%$ éxtasis y $6 \%$ heroína.

Los cambios en el Sistema Nervioso Central (SNC) generan tolerancia a la sustancia, caracterizada por la necesidad 
de incrementar la frecuencia y cantidad de droga para lograr un efecto de recompensa. La que en inicio fue de amplia magnitud, pero con el tiempo se transforma en experiencias desagradables $\mathrm{y}$ dolorosas (abstinencia). La compulsión y necesidad de no sentir el dolor de la abstinencia, lleva al sujeto a ingerir mayor cantidad en donde un $6 \%$ muere por sobredosis (NIDA, 2007a, 2010a).

Con el consumo, también se dañan órganos internos como el corazón, riñones, hígado que terminan produciendo cirrosis, deficiencias cardio-pulmonares, cáncer de pulmón y accidentes cerebro vasculares (NIDA, 2010a) trastornos neurológicos y psiquiátricos (esquizofrenia, síndrome de korsakoff, psicosis, tendencia suicida, síndrome a motivacional) (Minsalud, 2008; Pérez, et al. 2015).

Los costos por consumo de tabaco y alcohol es de 10.7 millones de dólares y por drogas ilegales es de 700.000 millones de dólares al año en Estados Unidos (López, 2012) sin contar las consecuencias sociales relacionadas como delincuencia $u$ otras, además de reducir o eliminar la actividad laboral y productiva de los estados en casi 59 millones por consumo de tabaco, 58 millones por alcohol y 20 millones por drogas, condicionantes medidos con el índice AVAD (Años de Vida Ajustados a Discapacidad) para el año 2000 en Estados Unidos (Martínez, Pérez y De las Heras, 2008).

\section{Error en el modelo de intervención (una crisis política)}

Es innegable el daño que producen las drogas; se reconoce un aumento en consumidores, lo que ha traído consecuencias individuales, sociales, políticas y económicas. La situación descrita ha llevado a los estados a definir políticas para su intervención, las cuales se interpretan desde una visión económica y basada en preceptos de oferta y demanda. La tesis de la oferta sostiene que al eliminar la producción terminaría la problemática y al contrario los teóricos de la demanda consideran que es necesario reducir la posibilidad de compra.

Ante la existencia de dos caminos para intervenir. Colombia y otros países han tomado la decisión de intervenir la oferta e incluir una política que elimina a productores y comerciantes y enfocándose en la tesis, que sin producto no hay oferta y sin oferta no hay demanda ni consumo (Thoumi, 2009). Uno de los ejemplos de esta propuesta es el Plan Colombia, desarrollada en convenio con los Estados Unidos y que hasta el año 2000 conto con recursos de 18.500 millones de dólares al año, más apoyo militar y permitiendo la creación de batallones especializados y la erradicación con glifosato. Entre sus resultados se cuentan miles de hectáreas destruidas y toneladas de droga incautada, encarcelamiento y muerte de carteles del narcotráfico ubicados en guerrillas, paramilitares y políticos del país (Morín y Collins, 2000 citados en Becoña, 2002; Pérez y Mejía; 2015).

Pero el Plan Colombia, también integro al país en una guerra civil enfrentando a narcotraficantes y militares de zonas urbanas y rurales por más de tres décadas, que como resultados ha dejo el aumento de la pobreza, desplazamiento y terrorismo (Thoumi, 2009) y con el tiempo se considera un plan fallido, ya que en la actualidad solo es posible incautar una de diez drogas que se producen y ha aumentado en el país consumidores y productores que involucra a nuevas mafias en todos los sectores como el político, económico y justicia (Pérez, Mejía y Becoña, 2015). 
El error en la intervención de la oferta ha llevado a diversos países a repensar el paradigma y cambiar sus preceptos que pasan de un concepto de represión a uno de legalización (Uribe y Castaño, 2014). En Colombia, el presidente Juan Manuel Santos, en el marco de la cumbre de los Estados Latinoamericanos realizada en Cartagena en 2012 ha nombrado la legalización de la marihuana (Pérez, Mejía y Becoña, 2015) y diversos países del continente como Bolivia o estados de Estados Unidos han compartido estas consignas.

La idea de la posible legalización de las drogas, como ha sucedido con el alcohol y tabaco modifican el paradigma de observación del fenómeno. No enfocándose en la oferta si no en el demandante o comprador. El comprador que es el individuo que realiza la interacción, con elementos externalizantes (compra) pero también internalizantes como búsqueda y elección. Convirtiendo al sujeto en responsable de la decisión y de mantener la oferta por su compra (Echavarría y Giraldo, 2015; Najul y Witzke, 2008; OMS, 2005; Papazian, Alfonso y Luzondo, 2006; Portellano, 2005).

El demandante de una droga un problema de tipo cognitivo, neuropsicológico y del desarrollo

Los estudios relacionados con las drogas y la conducta de consumo, parecieran mostrar una línea divisoria y ubicarse en dos momentos específicos. Uno antes y otro después de consumir la primera droga. Los estudios enfocados en explicar que sucede después de consumir la primera droga, han logrado profundizar sobre las consecuencias acontecidas en las estructuras neurológicas y neuropsicológicas provocadas por la toxicidad de la sustancia, además han identificado el origen de los trastornos clínicos por el consumo, abuso y dependencia a través de la explicación basada en la perversión de los sistemas dopaminergicos y su afectación en redes, conexiones con la corteza prefrontal y daños en las estructuras moleculares.

La segunda línea de investigación, se centra, en el antes de consumir, y basa sus preceptos en el intentar comprender factores causales que llevan a la decisión de consumir. Entre sus conclusiones están las de modelo sistémico que considera el acto de decidir y consumir, como un hecho atravesado por diversos factores como el político, económico, social, familiar, afectivo, personal y biológico, todos en igual importancia y todos con posibilidades de afectación en la conducta. Una de sus conclusiones más preponderantes son los factores de riesgo y protección.

Los factores de riesgo y protección, son un modelo que describe las relaciones existentes con las micro y macro estructuras del fenómeno, sin ánimo de ser concluyentes. Por ejemplo, se ha identificado que el consumo se asocia a condiciones de pobreza y ausencia de relaciones afectivas, sin embargo, existen sujetos con condiciones económicas y afectivas positivas, también en consumo, con diferencias en forma porcentual o uso de droga. Pero al final consumidores. Tal vez uno de los problemas que tiene este modelo se basa en un método que estudia relaciones entre procesos externos y consumo y no entre procesos subjetivos y consumo.

El factor subjetivo refiere a variables de estudio que describan las condiciones previas a la acción. Como lo propuesto por Ardila y Ostrosky (2008) quienes consideran que los individuos son capaces de realizar elecciones y conductas producto de sus representaciones y neuropsicología de la accion y es esto es porque lo sujetos son poseedores de lenguajes interiores 
caracterizados por ideas, pensamientos y representaciones, aprendidas en la interacción ambiental y el desarrollo de las funciones neurológicas del lenguaje.

El lenguaje interno que en principio fue externo, se expresa a través de contenidos semánticos, sintácticos y pragmáticos, los cuales son utilizados como recursos e instrumentos manipulables por las (FE). Estos que son producto de la corteza prefrontal y se desarrollan a través de los años y la madurez pero que sus formas de expresión dependen de la culturización y educación de los sujetos.

El lenguaje interno y su integración a las $\mathrm{FE}$, realizan acciones de planeación, memoria de trabajo, inhibición, toma de decisiones, entre otras y construyen una diversidad compleja de mundos posibles con relación ante un fenómeno. Su resultado expresado en lenguaje interior y con nicho en la $\mathrm{CPF}$, se proyecta a través de redes hacia áreas sensorio motoras en límites de la corteza frontal y parietal. La corteza sensorio motora primaria construye un mapa de acciones neurológicas que permite la activación del funcionamiento motriz y la secundaria realiza la acción, según lo definido por las estructuras mentales y neuropsicológicas (Martínez-Selva, Sánchez-Navarro, Bechara y Roman, 2006; Sastre-Riba, 2007).

En conclusión, la elección y acción de una conducta, son el producto de factores de tipo cognitivo expresados en representaciones, ideas o lenguajes internos, los que serían utilizados por las FE para realizar toma de decisiones, planeación, inhibición o flexibilidad, procedimientos que suceden en la CPF y se relacionan con la integración de redes neurológicas del lenguaje y su expresión en productos de la CPF, que logran desarrollarse en comportamientos gracias al área sensorio motriz que convierte las decisiones y su planeación en comportamientos con acción y movimiento.

Desde esta posición la elección de consumir o inhibir una droga tiene relación con las representaciones mentales y su interacción con las $\mathrm{FE}$, los cuales tomarían la decisión con mayor ventaja y llevando a la realización de procesos de planeación, fluidez o inhibición de la conducta. Pero si bien la elección y acción de consumo podrían tener relación con estos factores, se presentan nuevas preguntas reflexivas sobre la elección y acción como, ¿Por qué ante el estímulo (droga), los sujetos responden de forma diferente, algunos de forma positiva y otras negativas?, ¿unos inhiben y otros desarrollan acciones de consumo?, preguntas, que pueden ser explicadas desde las variables mencionadas.

Elección de consumo de drogas, propuesta cognitiva y neuropsicológica

En la psicología cognitiva las representaciones mentales o también definidas como pensamientos o ideas, son de alta importancia, en tanto se consideran estructuras mentales con capacidad para inhibir, definir y desarrollar una conducta. Incluso para este paradigma la cognición y la conducta son sinónimos al desarrollarse de forma continua y transversal. Algunos de sus autores como Bandura (aprendizaje vicario), Beck (ideas racionales - irracionales), Piaget (Constructivismo y desarrollo del pensamiento) (Sánchez, 2009) y Vygotsky (pensamiento y lenguaje) (Martínez et al., 2008; Torralba, 2015).

Dentro de este paradigma, emerge un concepto de importancia en la comprensión del antecedente comportamental y es la intencionalidad. La intencionalidad es una representación mental con contenido semántico que define y justifica la posibilidad o inhibición del desarrollo de una 
conducta o comportamiento y que según Ajzen citado por Martín, Martínez y Rojas (2011) esta estructura mental también puede ser definida como creencia.

Las creencias son ideas con contenidos favorables o no favorables hacia un objeto o conducta las cuales potencializan su posibilidad de acción. Según Ajzen (2011), estas creencias pueden ser de tres tipos. 1) creencias de actitudes con valoraciones positivas o negativas de un objeto $o$ realidad, 2) creencias de norma subjetiva con percepciones positivas o negativas de otros respecto al objeto o conducta y 3 ) creencias de control conductual percibido, sobre la existencia o deficiencia de recursos personales y ambientales para realizar una conducta. Según el autor el análisis de las tres creencias definirían la intención de una conducta (Luengo, Romero, Gómez, Guerra y Lence, 2012; Peltzer, Brandariz, Biscarra, Santángelo, Conde y Cremonte, 2013). La relaciones conductuales generan problemas de convivencia en el ámbito de influencia del sujeto y Robles (2015) habla de adquisición de patrones de comportamiento y aprendizaje social.

El modelo considerado con posibilidad de definir la intención predictiva hacia una conducta, ha sido denominado teoría de conducta planificada (Ajzen, 2011) y el cual goza de recomendaciones de la OMS y Organización Panamericana de la Salud (OPS) para su uso en forma de prevención en estudios de consumo de drogas, sexualidad o prevención de enfermedades (OMS, 2005; OPS citado en Arbaiza, 2014; Tort, Cortes, Gimenez, Luque y Gomez, 2011; Peltzer, et al. 2013; Lloret, Morell-Gomis, Laguia y Moriano, 2017).

En el fenómeno de elección y consumo de drogas, la utilización de un modelo como la teoría de conducta planificada permite describir los contenidos menta- les sobre las drogas y además los tipos de creencias según actitud, norma subjetiva, control conductual e intencionalidad, que tienen los sujetos y los cuales según el modelo neuropsicológico servirían de recursos para el procesamiento de las FE. Es necesario mencionar que los contenidos y complejidad de las creencias e intencionalidad, no son similares en los sujetos, ya que estos dependen de los tipos de experiencias acontecidos en los sujetos (constructivismo).

Por su parte, las FE son un factor de tipo neuropsicológico que explica cómo se desarrollan los procesos neurológicos y su funcionalidad a través de procesos superiores como la atención - concentración, memoria de trabajo -MT- (sostener, controlar y elegir información), flexibilidad mental (Stelzer, Cervigni y Martino, 2010) fluidez verbal (Tirapu-Ustarroz, Muñoz-Céspedes y Pelegrin-Valero, 2002) toma de decisión, inhibición y planeación.

Un ejemplo es la toma de decisiones, que es un factor neuropsicológico resultado de la acción entre el sistema límbico y la CPF con interacción de NT como la dopamina, serotonina y otros, representados en la acción para elegir una alternativa entre un rango de diversas propuestas (Bausela, 2007) su elección ofrecería una ventaja presente y futura (Verdejo, 2006; Oliva, 2007) pero su existencia no garantiza la elección de estímulos siempre con ventaja, ya que esta depende de procesos de madurez que involucran mecanismos biológicos y culturales.

Damazio y Bechara citados en De Lorenzo (2012) identificaron que los pacientes con daños neurológicos en la corteza orbito frontal o sistema límbico manifiestan tendencia a realizar elecciones impulsivas, de riesgo, con desventaja o sin identificación de peligros. Decisiones y comportamientos frecuentes en sujetos 
con consumo de drogas (O'Donoghue \& Rabin, 2000; Villalba y Verdejo-Garcia, 2012) y en menor magnitud en adolescentes, explicado por la inmadurez de su edad, la cual está en proceso de desarrollo, integración de redes y organización de regiones orbito frontal, ventromedial y dorso lateral que concluye en la segunda década de vida, con influencia por los procesos culturales como la educación y pautas de crianza (Asensio, 2011; Caña, Michilini, Acuña y Godoy, 2015).

La diferencia en la madurez de los sujetos debido a su desarrollo biológico y cambios en la estimulación cultural, definiría las diferencias en las formas de desarrollar el funcionamiento ejecutivo y sus productos, lo cual explicaría por qué las diferencias en las formas de elección y planeación ante un mismo estimulo como la droga entre los adolescentes.

Otras funciones ejecutivas implicadas son la planeación e inhibición. La primera se describe como la capacidad para generar programas meta de acción presente y futura y la inhibición como el proceso que permite controlar, regular e inhibir información sensorial, cognitiva o neurológica, que pudieran interferir el proceso. Las funciones de planeación e inhibición son las que después de la decisión, estructuran un plan bien sea para gestionar, buscar y desarrollar la acción de compra o para inhibirla.

Al igual que en la toma de decisión, la planeación e inhibición en los seres humanos presentan formas diferentes de desarrollarse, esto no significa que no existan pero su forma de modulación y ejecución se transforma dependiendo de las condiciones biológicas y madurez (Verdejo, 2006) un ejemplo son los sujetos con Trastornos de Déficit de Atención e Hiperactividad (TDAH), adicción, comportamiento impulsivo, negativista - desafiante o disocial que puntúan en las pruebas de funcionamiento ejecutivo más bajo que los sujetos que no presentan estas deficiencias comportamentales (Florez, Castillo y Jimenez, 2014; Florez y Ostrosky, 2012).

Incluso los individuos sin aparentes daños neurológicos, pero con trastornos de personalidad, bipolar, depresión o hijos de adictos, presentan diferencias en el funcionamiento ejecutivo, no mostrando patologías, pero si deficiencias en el desarrollo ejecutivo (Rivarola, Pilatti, Godoy, Brussino y Pautassi, 2016). También en la adolescencia y como producto de la inmadurez en el desarrollo de la CPF los sujetos tendrían tendencia a mostrar deficiencias para la planeación e inhibición y de otras funciones ejecutivas (TirapuUstarroz, Muñoz-Cespedes y Pelegrin-Valero, 2002; Verdejo y Perez-Garcia, 2005; Vivo, Saric, Muñoz, McCoy, López-Peña y Bautista-Arredondo, 2013) lo cual explicaría la heterogeneidad en la forma de realizar elecciones de consumo y su posterior acción.

¿Por qué la elección y consumo de drogas en la adolescencia?, hipótesis explicativa

Los estudios buscan definir los factores intervinientes en la decisión del consumo de una droga en la adolescencia, presentan importancia en tanto el $16 \%$ de sujetos inician el consumo en esta etapa e incluso la OMS la ha definido una enfermedad del desarrollo (NIDA, 2010b; Ministerio de protección social y salud 2008; Martinez y Ojeda, 2016; Méndez, 2012) nombrándose como un hecho preocupante en tanto para el 2009 en América Latina existían 150 millones de personas entre los 10 a 24 años, casi el $28 \%$ de la población completamente vulnerables y en una época en la que no existe ni el conocimiento, ni la política para prevenir o 
intervenir la pandemia con confiabilidad (Comisión Económica para América Latina y el Caribe-CEPAL, citado en Vivo, et al. 2013).

Este es un hecho consecuente, en tanto para el adolescente, esta etapa presenta cierta dificultades incontrolables, como lo es la activación hormonal acompañada de transformaciones físicas, desarrollo sexual y deseante de una pareja y cambios psicológicos que implican el camino hacia la independencia e identidad, junto con el acoplamiento a estructuras y entramados sociales que exigen cierta individualidad y al mismo tiempo una cohesión de grupo entre pares que excluye y distancia al sistema familiar.

Son múltiples los aspectos que debe resolver el adolescente y el cual no cuenta con recursos suficientes de tipo neuropsicológico para permitirle una mejor coherencia en la toma de decisiones, planeación o inhibición de su intencionalidad. Condiciones que lo hacen vulnerable ante un entramado biológico, psicológico y social que le exige una forma de interpretar y resolver la realidad, llenándolo de incertidumbre y conflicto (Luengo, et al. 2012).

Pero el hecho de encontrarse en incertidumbre o conflicto, no le impiden tener la responsabilidad de tomar decisiones y realizar sistemas de planeación e inhibición. Tal vez el adolescente con contenidos de creencias e intencionalidad negativa hacia las drogas y el consumo y con positiva maduración de las $\mathrm{FE}$, podrían sortear la elección y lograr respuestas negativas ante el consumo, inhibiendo este comportamiento y activando otras estrategias de fluidez para una planeación diferencial.

Pero el adolescente con contenidos de creencias e intencionalidad positiva hacia las drogas e inmadurez en las FE posiblemente elegiría el consumir una droga de forma impulsiva con recompensa actual, pero con desventaja futura (algunos reconocen las consecuencias, pero asumen la recompensa inmediata de mayor ventaja que la futura) organizando planes de corto y no de largo plazo e inhibiría acciones que no le permitan llegar a su cometido.

La elección positiva y posterior gestión de consumo en el adolescente, en muchos casos lleva a cuadros clínicos de dependencia y abuso. Algunos desertan de la escuela producto del bajo rendimiento o fracaso escolar, otros abandonan sus casas para continuar periodos profundos de consumo que se prolongan por tiempos largos, acompañados de actividades delictivas y de calle (Cookea, Dahdaha, Normanb y Frenchc, 2016; Melo y Casthaneria, 2010; OMS, 2005; Pérez, Mejía y Becoña, 2015). En algunos casos el adolescente sin recursos neuropsicológicos, no realizaría la elección tras su análisis respectivo, sino que, ante la presión por una decisión, terminaría realizando una elección promovida por el ambiente como pares, jibaros o medios de comunicación y no por otros referentes como padres, ya que estos últimos ofrecen recompensas futuras, pero no inmediatas.

\section{Conclusiones}

El consumo de drogas y adicción en la adolescencia es un fenómeno objetivo con una tasa de crecimiento superior al $60 \%$ en países de América Latina. Su acción y consecuencias ha llevado a definirse como una pandemia y enfermedad del desarrollo y la que actualmente es de difícil promoción, prevención e intervención.

Las formas de intervención de tipo político y económico que se centra en la eliminación de la oferta y el control de la producción. Son en su gran mayoría ineficientes y se requiere de programas y políticas públicas con enfoque en el análisis y comprensión del fenómeno de la demanda y el sujeto 
comprador. El cual implique la explicación por las formas de decisión y acción de una conducta de consumo de drogas.

La elección y realización de conducta de consumo, puede ser explicado desde tres variables de análisis. La primera referida a factores cognitivos de intencionalidad y creencias el cual implica sus contenidos y complejidad, la segunda son los factores neuropsicológicos como las FE las cuales utilizan las estructuras cognitivas para la toma de decisiones y posterior planeación, fluidez e inhibición y por ultimo está el factor de desarrollo y madurez, la cual explica que en la adolescencia, los recursos neuropsicológicos a un están en organización de ahí la alta vulnerabilidad por realizar elecciones impulsivas y con desventaja futura.

\section{REFERENCIAS}

Ajzen, I. (2011). The theory of planned behaviour: Reactions and reflections. Psychology \& Health, 26(9). 11131127. https://doi.org/10.1080/088704 46.2011.613995

APA. (2014). Guia de consulta de los criterios diagnosticos del DSM-5. Washington, D.C.: American Psychiatric Publishing.

Andrade, P., Betancourt, D., Moreno, N. y Alvis, L. (2017). Fortalezas externas desde el modelo de desarrollo positivo de los jóvenes y consumo de sustancias en una muestra de adolescentes mexicanos y colombianos. Avances en Psicología Latinoamericana, 35(3). 515-529. http://dx.doi. org/10.12804/revistas.urosario.edu. co/apl/a.4095

Arbaiza, M. (2014). Alteraciones cognitivas, conectividad funcional y personalidad en el drogodependiente. [Tesis doctoral]. Universidad complutense, Madrid, España.
Ardila, A. y Ostrosky, F. (2008). Desarrollo histórico de las funciones ejecutivas. Revista de neuropsicología, neuropsiquiatria y neurociencias, 8(1). 1-21.

Asensio, S. (2011). Bases neuroanatomicas y neurofuncionales del trastorno de abuso de alcohol y su relación con la impulsividad: estudio mediante resonancia magnética. [Tesis doctoral]. Universidad Cardenal Herrera, Valencia, España.

Bausela, E. (2007). Implicaciones de las conexiones cortico y subcorticales del lóbulo frontal en la conducta humana. Docencia e Investigación, 32(17).7-24.

Becoña E. (2002). Bases científicas de la prevención de las drogodependencias. Madrid: Plan Nacional sobre Drogas.

Buela-Casal, G., Riaño-Hernandez, D. y Guillen, A. (2015). Conceptualización y evaluación de la impulsividad en adolescentes: una revisión sistemática. Universitas Psychologica, 14(3). 1077-1090. http://dx.doi.org/10.11144/ Javeriana.upsy14-3.ceia

Caña, M., Michilini, Y., Acuña, G. y Godoy, J. (2015). Efectos de la impulsividad y el consumo de alcohol sobre la toma de decisiones en los adolescentes. Health and adicctions, 15(1). 55-66.

Castellanos, J. y Espinosa, G. (2013). Revisión de las tendencias de investigación sobre consumo de sustancias ilegales por los jóvenes. Virajes, 15(2). 57-71.

Corominas, M., Roncero, C., Bruguera, E. y Casas, M. (2007). Sistema dopaminergico y adicciones. Revista Neurologia, 44(1). 23-31.

Cookea, R., Dahdaha, M., Normanb, P. y Frenchc, D. (2016). How well does the theory of planned behaviour predict alcohol consumption? A systematic review and meta-analysis. Health Psychology Review, 10(2). 148-167. http://dx.doi.org/10.1080/17437199.20 14.947547 
De Lorenzo, D. (2012). La comprensión de las bases biológicas de la personalidad en el siglo XXI: genómica de la impulsividad. En J. Celma y F. Abella (Edit.), Neuropsicología de la impulsividad. Actualizaciones. Lleida: Universidad de Lleida.

Echavarria, M. y Giraldo, A. (2015). Abordaje integral para la intervención de las conductas adictivas. Manual practico para la detección, diagnostico, remisión y tratamiento en el marco de la atención primaria en salud. Medellín: Fundacion Universitaria Luis Amigo.

Florez, J., Castillo, R., y Jimenez, N. (2014). Desarrollo de las funciones ejecutivas, de la niñez a la juventud. Anales de psicología, 30(2). 463473.

Florez, J. y Ostrosky, F. (2012). Desarrollo neuropsicológico de lobulos frontales y funciones ejecutivas. México, D.F.: Manual Moderno.

Fuenmayor, D. y Saavedra, B. (2015). Síndrome disejecutivo en adolescentes en conflicto con ley penal. [Tesis de grado]. Universidad Rafael Urdaneta, Maracaibo, Venezuela.

Hidalgo, M. y Redondo, A. (2005). Consumo de drogas en la adolescencia. Pediatric Integral, 9(1). 137-155.

López, M. (2012). Determinantes de la conducta planeada y el significado del consumo de tabaco y/o alcohol $y$ el consumo de estas sustancias en adolescentes. [Tesis doctoral]Universidad de Nuevo León, México.

Luengo, M., Romero, E., Gómez, J., Guerra, A. y Lence, M. (2012). La prevencion del consumo de drogas y la conducta antisocial en la escuela: análisis y evaluación de un programa. Santiago de Compostela: Universidad de Santiago de Compostela.
Lloret, D., Morell-Gomis, R., Laguia, A. y Moriano, J. (2017). Diseño y validación de una escala de consumo de cannabis (Quic) para adolescentes. Adicciones, 30(1). 11-12.

Martín, M, Martínez, J. y Rojas, D. (2011). Teoría del comportamiento planificado y conducta sexual de riesgo en hombres homosexuales. Revista Panam Salud Publica. 29(6). 433-43.

Martínez, I. (2012). Efectos de la exposicion y calidad de la prevencion escolar en el consumo de drogas y factores de riesgo en estudiantes de secundaria. [Tesis doctoral]. Universidad complutense de Madrid, España.

Martínez, L. y Ojeda, T. (2016). Informe sobre habitos de consumo de drogas entre adolescentes y jóvenes en espacios de ocio: resultados de intervención de una noche de fiesta. Unidad de prevencion comunitaria en conductas adictivas. Ayuntamientos de Alfanfar. España.

Martínez, F., Pérez, E. y De las Heras, M. (2008). Nexus. Programa para la prevencion del consumo de drogas. Madrid: Deporte y vida. Recuperado en: http://www.fundacioncsz.org/ ArchivosPublicaciones/166.pdf

Martínez-Selva, J., Sánchez-Navarro, J., Bechara, J. y Roman, F. (2006). Mecanismos cerebrales de la toma de decisiones. Revista de neurología, 42(7). 411-418. Recuperado de https://doi.org/10.33588/ rn.4207.2006161

Méndez, M. (2012). Variables de conducta, factores de riesgo para la salud $y$ adaptación integral relacionados con la problemática bullying en estudiantes de educacion secundaria. [Tesis doctoral]. Universidad de Murcial, España. 
Melo, D. y Casthaneria, L. (2010). Autoeficacia y actitud hacia el consumo de drogas en la infancia: explorando los conceptos. Revista Latino-am Enfermagen. 18(spec.) 658-662.

NIDA. (2015). La marihuana. [Reporte].Recuperado de https://d14rmgtrwzf5a.cloudfront.net/sites/default/ files/1832-la-marihuana.pdf

NIDA. (2012). Los medicamentos de prescripción: abuso y adicción. [Reporte]. Recuperado de https://d14rmgtrwzf5a.cloudfront.net/sites/default/ files/prescriptiondrugs_rrs_sp_1.pdf

NIDA. (2011). Abuso de inhalantes. [Reporte]. Recuperado de https://www. drugabuse.gov/es/publicaciones/ serie-de-reportes/abuso-de-inhalantes/nota-de-la-directora

NIDA. (2010a). Adiccion al tabaco. [Reporte]. Recuperado de https://d14rmgtrwzf5a.cloudfront.net/sites/default/files/rrtabaco_0.pdf

NIDA. (2010b). Cocaína abuso y adicción. [Reporte]. Recuperado de https:// d14rmgtrwzf5a.cloudfront.net/sites/ default/files/rrcocaina.pdf

NIDA. (2007a). Abuso de los esteroides anabólicos. [Reporte]. Recuperado de https://d14rmgtrwzf5a.cloudfront. net/sites/default/files/esteroides.pdf

NIDA. (2007b). Abuso y adicción a la metanfetamina. [Reporte]. Recuperado de https://d14rmgtrwzf5a. cloudfront.net/sites/default/files/rrmetanfeta.pdf

Najul, R. y Witzke. M. (2008). Funciones ejecutivas y desarrollo humano y comunitario. Kaleidoscopio, 5(9). $58-74$

O'Donoghue, T. \& Rabin, M. (2000). The economics of immediaty gratificaction. Journal of behavioral decisión making. 13(2). 233-250. https://doi.org/10.1002/(SICI)10990771(200004/06)13:2<233::AID BDM325>3.0.CO;2-U
Oliva, A. (2007). Desarrollo cerebral y asunción de riesgos durante la adolescencia. Apuntes de psicología, 25(3). 239-254.

OMS. (2005). Neurociencia del consumo y dependencia de sustancias psicoactivas. Suiza: OMS.

Papazian, O., Alfonso, I. y Luzondo, R. (2006). Trastornos de las funciones ejecutivas. Revista de neurología, 42(supl. 3). S45-s50

Patiño, J. (2012). Patrones de consumo de cocaina en los jóvenes. [Tesis doctoral. Universidad de Girona, España.

Peltzer, R., Brandariz, R., Biscarra, M., Santángelo, P., Conde, K. y Cremonte, M. (2013). Predictores de la intención se consumo intensivo episódico de alcohol en adolescentes. Temas en psicología, 21(1). 183-192. Recuperado de http://hdl. handle.net/11336/3294

Pérez, A., Mejía, J. y Becoña, E. (2015). De la prevención y otras historias. historia y evolución de la prevención del consumo de alcohol y drogas en américa latina y en Europa. Bogotá, D.C.: California Edit. Recuperado de http://www.irefrea.eu/uploads/ PDF/Perez-etal_Ed_Prevencion-yotras-historias_2015.pdf

Pérez, A., Mejía, J., Reyes, M. y Cardozo, F. (2015). Consumo de alcohol en menores de 18 años en Colombia: 2015. Segundo estudio en siete capitales y dos municipios pequeños. Bogotá, D.C.: Coorporación nuevo rumbo. Recuperado de http:// nuevosrumbos.org/wp-content/ uploads/2015/12/Consumo-de-Alcohol-en-menores-de-edad-en-Colombia-2015.pdf

Portellano, J. (2005). Como desarrollar la inteligencia. Entrenamiento neuropsicológico de la atención y funciones ejecutivas. Madrid: SomosPsicología. 
Quintero, M. (2015). La prevencion de las adicciones en contextos familiares $y$ escolares: análisis del programa de prevencion de la asociación proyecto hombre "entre todos". [Tesis doctoral]. Universidad de Granada, España.

República de Colombia. Minsalud (2007). Política nacional para la reducción del consumo de sustancias psicoactivas y su impacto. Recuperado de http://www.odc.gov.co/Portals/1/ Docs/politDrogas/politica_nacional_ consumo.pdf

Robles, A. (2015). Propuesta de convivencia escolar a través de talleres de prevención de violencia escolar con perspectiva de género. Cultura, Educación y Sociedad, 6(2). 29-49. Recuperado de https://revistascientificas.cuc.edu. co/culturaeducacionysociedad/article/ view/841

Ruiz-Sánchez, J., Pedrero-Pérez, E., Lozoya-Delgado, P., Llanero-Luque, M., Rojo-Mota, G. y Puerta-García, C. (2012). Inventario de síntomas prefrontales para la evaluación clínica de las adicciones en la vida diaria: proceso de creación y propiedades psicométricas. Revista de neurología, 54(11). 649-663.

Rivarola, G., Pilatti, A., Godoy, J., Brussino, S. y Pautassi, R. (2016). Modelo de predisposición adquirida para el uso de alcohol en adolescentes argentinos. Suma psicológica. 23.(2). 116-124. http://dx.doi.org/10.1016/j. sumpsi.2016.03.002

Sánchez, L. (2009). Las percepciones sociales como determinantes del consumo de psicoestimulantes entre los jóvenes riojanos. Logroño: Observatorio Riojano sobre drogas.

Sánchez, T., Arroyo, A. y Azcona, A. (2014). Relacion entre consumo de sustancias, busqueda de sensaciones, impulsividad y síndrome disejecutivo en población universitaria. Reduca, 6(4). 312-321.
Sastre-Riba, S. (2006). Condiciones tempranas del desarrollo y el aprendizaje: el papel de las funciones ejecutivas. Revista de neurología. 46(suple. 2). S143-151. https://doi. org/10.33588/rn.42S02.2005782

Stelzer, F., Cervigni, M. y Martino, P. (2010). Bases neurales del desarrollo de las funciones ejecutivas durante la infancia y la adolescencia. Una revisión. Revista chilena de neuropsicología. 5(3). 176-184.

Thoumi (2009). Una mirada regional: la economía de las drogas en America Latina. En, Colectivo Maloka (Ed.), La economía de las drogas ilícitas. Escenarios de conflicto y derechos humanos. (54-84). Barcelona: Cidob.

Tirapu-Ustarroz, J., Muñoz-Cespedes, J. y Pelegrin-Valero, C. (2002). Funciones ejecutivas. Necesidad de una integración conceptual. Revista de neurología, 34(7). 673-685

Torralba, E. (2015). Estudio psicosocial de las actividades de ocio en la adolescencia. [Tesis doctoral]. Universidad de Castilla, La Mancha, España.

Toaza, A. (2011). Percepción de riesgos asociados al consumo de drogas $y$ su influencia en estudiantes de octavo año de básica y primero de bachillerato de los colegios Padre Miguel Gamboa. [Trabajo de grado]. Universidad Central, Quito, Ecuador.

Tort, B., Cortes, M., Gimenez, J., Luque, L. y Gomez, R. (2011). Elaboración de un cuestionario basado en la teoría de conducta planificada de Ajzen para evaluar el consumo de alcohol en atrancón en adolescentes. Revis ta española de drogodependencia, 36(4). 403-412.

Uribe, N. y Castaño, G. (2014). Barras de futbol, consumo de drogas y violencia. Psicología desde el caribe, 31(2), 243-279. 
Vivo, S., Saric, D., Muñoz, R., McCoy, S., López-Peña- P. y Bautista-Arredondo, S. (2013). Guía para medir comportamiento de riesgo en jóvenes. Corea/ Finlandia: BID.

Verdejo, A. (2006). Funciones ejecutivas y toma de decisiones en drogodependientes: rendimiento neuropsicológico y funcionamiento cerebral. [Tesis doctoral]. Universidad de Granada, España.

Verdejo, A. y Perez-Garcia, M. (2005). Neuropsicologia en el ámbito de las drogodependencias. Evaluación de las funciones ejecutivas. Proyecto Hombre, 53(1). 39-43.

Villalba, E. y Verdejo-Garcia, A. (2012). Procesamiento emocional, interocepcion y funciones ejecutivas en policonsumidores de drogas en tratamiento. Trastornos adictivos, 14(1). 10-20.
Oscar Armando Erazo Santander es Psicologo, especialista en Intervención Social y Problemas Humanos con master en Neuropsicología y Educación, a realizado cursos doctorales en ciencias sociales, niñez y juventud en la Universidad de Manizales (Colombia) y es candidato a Doctor en Psicologia orientación en neurociencias cognitivas aplicadas de la Universidad Maimonides (Argentina). 